http://jmscr.igmpublication.org/home/ ISSN (e)-2347-176x ISSN (p) 2455-0450 crossref DOI: https://dx.doi.org/10.18535/jmscr/v8i2.22

\title{
A Case Report of Mucopolysaccharidosis Type 1 (MPS I H - Hurler Syndrome)
}

\author{
Authors \\ Dr Kadiyala Meena ${ }^{1}$, Dr Boyapati Srilakshmi ${ }^{2}$, Dr Hinduja Reddipilli ${ }^{3}$, \\ Dr P.Yashodhara ${ }^{4}$, Dr Jhansi Rani ${ }^{5}$ \\ ${ }_{1,2,3}$ Residents, ${ }^{4}$ Professor, ${ }^{5}$ Associate Professor \\ Department of Paediatrics, Guntur Medical College, Guntur, Andhra Pradesh, India, 522004 \\ *Corresponding Author \\ Dr Boyapati Srilakshmi
}

\begin{abstract}
Mucopolysaccharidosis type 1 (MPS IH, Hurler syndrome) is a rare genetic disorder caused by deficiency of enzyme $\alpha-L$ - iduronidase, which is needed for degradation of glycosaminoglycans (GAG) (dermatansulfate \& heparansulfate). Incomplete degradation of these substances, resulting in intralysosomal accumulation of glycosaminoglycan fragments. The distended lysosomes accumulate in the cell in various organs of the body, interfere with cell function \& lead to the characteristic pattern of clinical, radiological, and biochemical abnormalities ${ }^{(1,3)}$. Deficiency of $\alpha$-L-iduronidase results in a wide range of clinical involvement from severe Hurler disease to mild Scheiedisease (MPS I S) \& intermediate form Hurler-Scheie disease (MPS I HS). Hurler syndrome is a progressive disease which if left untreated, results in mental \& physical deterioration leading to significant morbidity \& mortality ${ }^{(1)}$. There are very few cases of Hurler syndrome reported in India. Here we are presenting an eight-year-old boy with a combination of clinical, radiological \& biochemical features confirmed as a case of Hurler syndrome.

Keywords: Mucopolysaccharidosis, Hurler syndrome, Scheiedisease, Glycosaminoglycans, dermatansulfate, heparansulfate.
\end{abstract}

\section{Introduction}

Mucopolysaccharidoses are a group of rare genetic disorders caused by the deficiency of lysosomal enzymes that catabolize glycosaminoglycans. All the mucopolysaccharidoses are inherited in an autosomal recessive pattern except Hunter disease

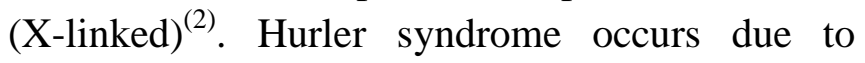
mutation in the IDUA gene mapped to the chromosome $4 \mathrm{p} 16.3$ encoding for $\alpha$-Liduronidase (1). It is the most common and severe form of mucopolysaccharidoses. The incidence of
MPS IH has been reported to be 1:1,00,000 per childbirth. MPS I has wide phenotypic variation, partly determined by residual enzyme level and the mutant allele.

\section{Case Report}

An 8-year-old male child born out of third-degree consanguineous marriage, first in birth order, was brought to pediatrics department with the chief complaint of seizures of 2days duration and intellectual disability with associated skeletal deformities observed since early childhood 
(approximately 1year of age). There was no significant perinatal history, and the younger sibling was healthy. The child had low intelligence quotient with speech disability and mild hearing loss. On examination (Fig 1) child had coarse facial features with a large head, prominent forehead, flat nasal bridge, broad nasal tip, large tongue, and short stature, multiple skeletal deformities with joint contractures, mild corneal clouding, hepatomegaly, splenomegaly.

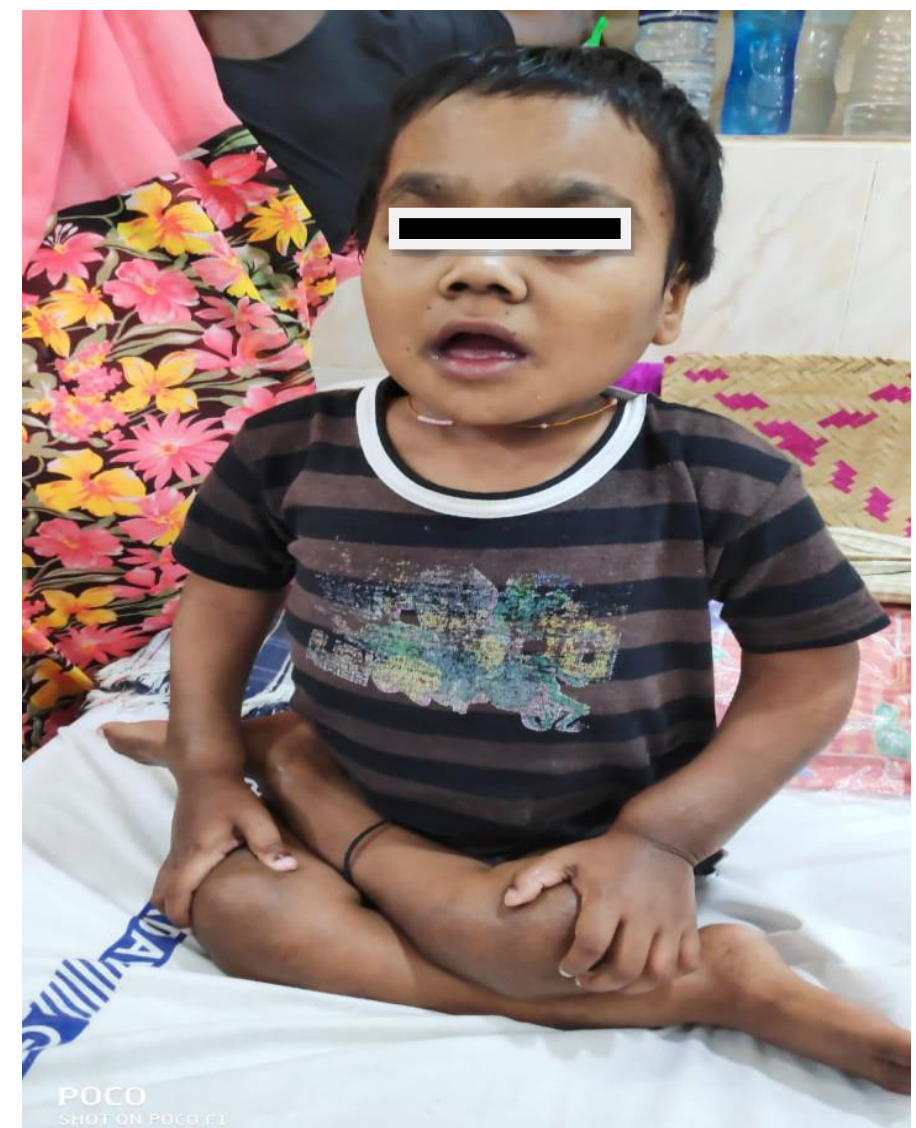

Fig 1: child with large head, corase facial features, short thick hands, joint contractures

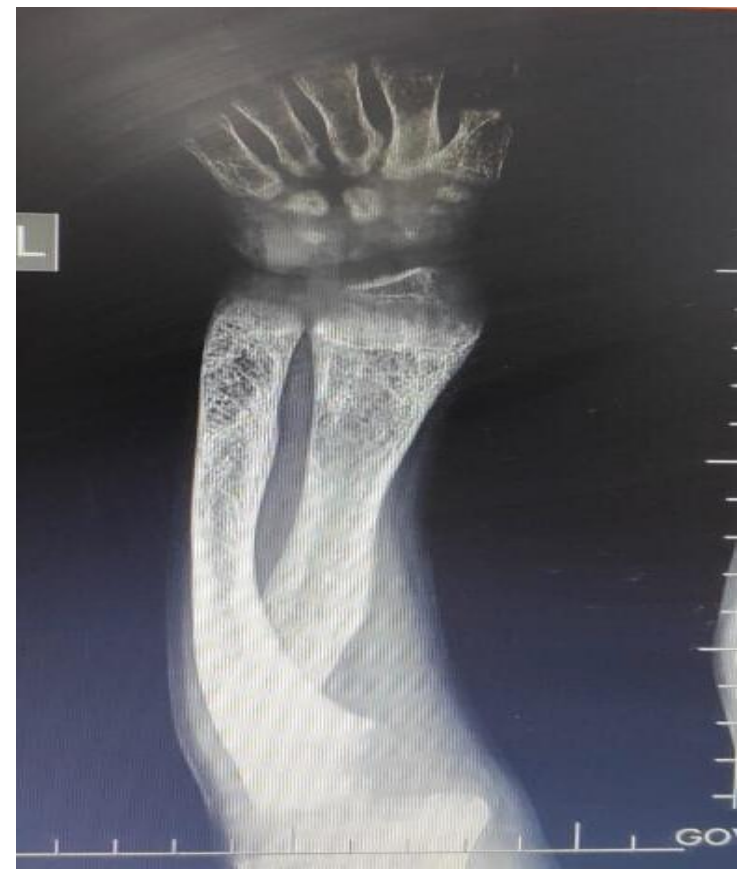

Fig 2: x-ray forearm with hand

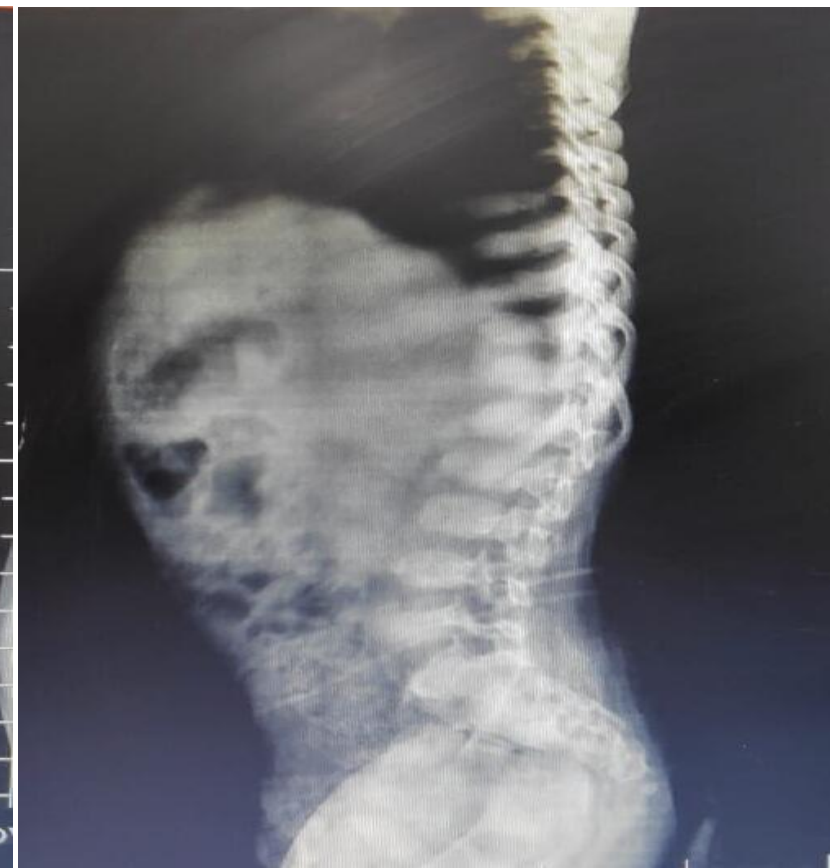

Fig 3: $x$-ray spine lateral view 


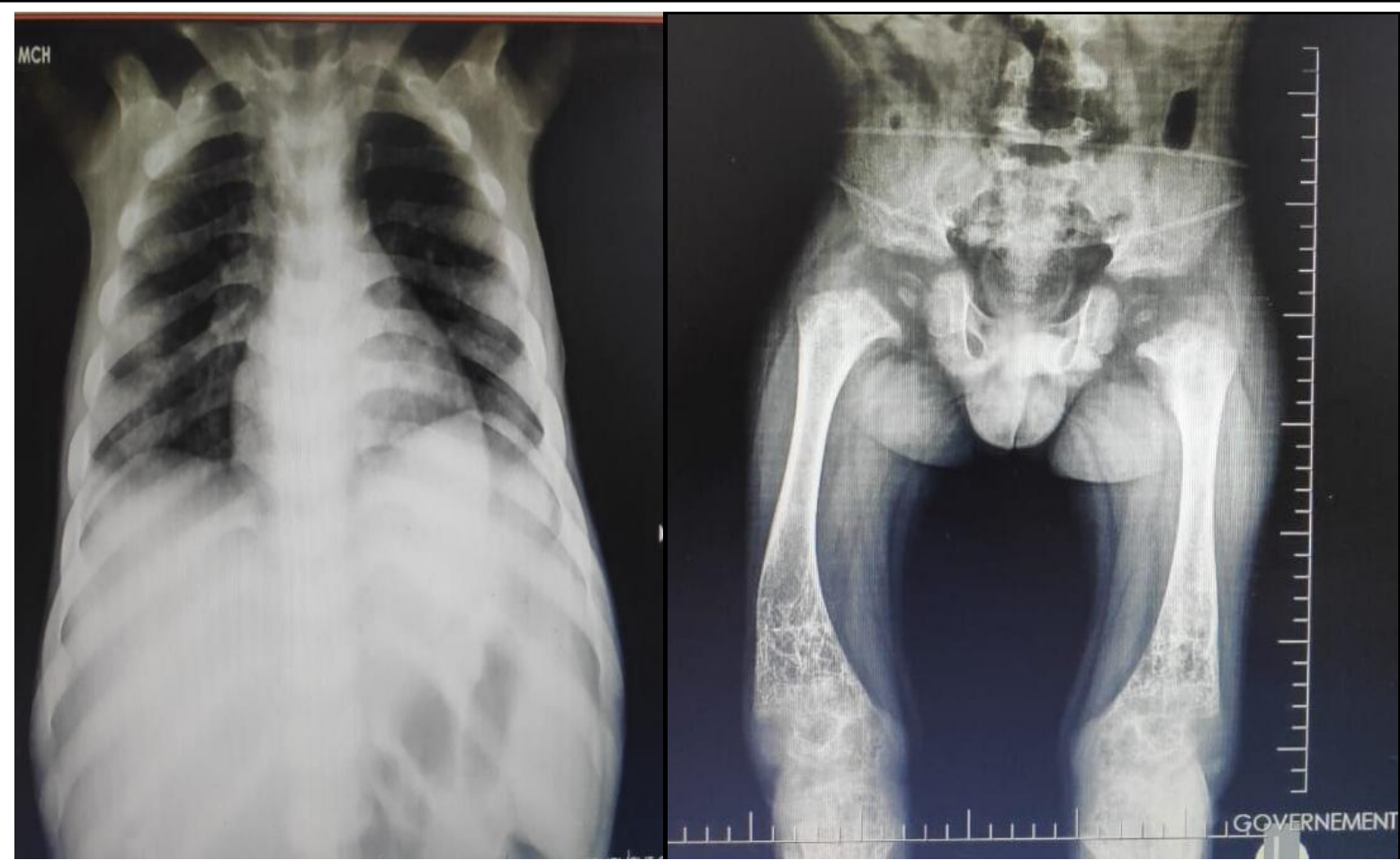

Fig 4: x-ray chest

Fig 5: x-ray pelvis with lower limbs

On investigating, the child had a mild degree of microcytic hypochromic anemia and hypothyroidism. Radiographical examination (Fig $2,3,4,5)$ revealed characteristic skeletal dysplasia (Dysostosis multiplex).

Fig 2: X-ray hand revealed delayed appearance of carpal bones with short and broad metaphysis \& proximal narrowing of the metaphysis. X-ray forearm showed widened diaphysis in both radius and ulna.

Fig 3: X-ray spine revealed anterior beaking of vertebral bodies

Fig 4: X-ray chest showed broad oar shaped ribs.

Fig 5: X-ray pelvis revealed narrowing of the lower part of the pelvis with shallow acetabular fossa. X-ray of lower limbs showed widened diaphysis of both femurs with a coarse trabecular pattern.

Echocardiogram showed mild mitral regurgitation with the thickened mitral valve. MRI brain revealed mild hydrocephalus.

Because of specific clinical and radiological features, mucopolysaccharidosis type 1 was suspected, and for confirmation of diagnosis, enzyme assay was sent, which showed low levels of $\alpha$-L- iduronidase, i.e., $1.05 \mathrm{nmol} / \mathrm{hr} / \mathrm{ml}$ (normal range was $2.4-12 \mathrm{nmol} / \mathrm{hr} / \mathrm{ml})$. Urinary excretion of glycosaminoglycans was not done. The child was stabilized and started on anti-epileptics for control of seizures. The child developed pneumonia during the hospital stay and was treated. Later, Child was sent to a higher center for further genetic analysis and enzyme replacement therapy.

\section{Discussion}

Hurler disease is a severe, progressive disorder with multiple organ and tissue involvement that results in premature death usually within 1st decade if left untreated as a result of cardio respiratory failure and progressive neurological disease $^{(3)}$. Children with Hurler syndrome are normal at birth except for inguinal hernia and are usually diagnosed between 6 months to 24 months of age ${ }^{(1)}$. They have a variable degree of growth failure, intellectual disability, coarse facial features, joint contractures, organomegaly, corneal clouding, dysostosis multiplex, glycosaminoglycanuria ${ }^{(2)}$.

Children with hurler syndrome also have a loss of vision (due to pigmentary retinopathy), hearing loss, otitis media, obstructive sleep apnea, 
recurrent respiratory infections. Cardiac involvement results in regurgitation across valves, cardiomyopathy, arrhythmias, and requires cardiac evaluation every 1 or 2 years after initial diagnosis $^{(3)}$. Regular dental and radiological assessment should be performed every six months and parents or caregivers should be educated about dental home care ${ }^{(4,5)}$. Hydrocephalus and spinal cord compression can also occur. Children with MPS I Hurler-Scheie have a later age at onset, usually in the second half of 1st decade and second decade or beyond for MPS I Scheie. Children with Scheie disease have preserved intellectual functioning ${ }^{(2)}$.

Early diagnosis and intervention can improve the life expectancy and quality of life in these children. A multidisciplinary approach is most crucial in the management of these patients and includes regular assessments, supportive care, and treatment of a variety of systemic complications $^{(6,7)}$. Hematopoietic stem cell therapy (HSCT) and enzyme replacement therapy (ERT) are currently available definitive treatment options along with providing supportive care ${ }^{(8)}$. HSCT if done early $(<2 \mathrm{yrs})$, can halt the progression of disease \& improves life expectancy, quality of life, growth, respiratory symptoms, visceromegaly, joint mobility, hearing, vision $^{(10)}$. Enzyme activity in serum and urinary GAG excretion normalizes. HSCT does not significantly improve the neuropsychological outcome in patients with impaired cognition at the time of presentation $^{(1)}$.

ERT using recombinant $\alpha$ - L- iduronidase (Aldurazyme) has been approved in MPS-I. The enzyme does not cross the blood-brain barrier \& does not prevent deterioration of cognition and other neurological functions. It is often instituted before stem cell transplant, and both may be combined for a better outcome ${ }^{(9)}$. Newer approaches such as intrathecal ERT, ERT with fusion proteins to cross the $\mathrm{BBB}$, gene therapy, substrate reduction therapy (SRT), chaperone therapy, and some combination of these strategies may provide better outcome for MPS patients in the near future ${ }^{(11)}$.

Primary prevention through genetic counseling and tertiary prevention to avoid or arrest complications remain the mainstay of pediatric care. Newborn screening methods by tandem mass spectrometry are available recently for early diagnosis of the disease so that treatment can be initiated early ${ }^{(12)}$.

\section{Financial support- none}

\section{Bibliography}

1. Nelson textbook of paediatrics $21^{\text {st }}$ edition Elsevier publication

2. PG textbook of paediatrics $2^{\text {nd }}$ edition JP publication

3. Neufeld EF, Muenzer J. The mucopolysaccharidoses. In: Scriver C, Beaudet A, Sly W, et al., editors. The Metabolic and Molecular Bases of Inherited Disease. New York NY: McGraw Hill; 2001. pp. 3421-52.

4. Mucopolysaccharidosis type I HurlerScheie syndrome: A rare case report Contemporary Clinical Dentistry. Jan-Mar 2011; 2(1)66

5. Muenzer J, Wraith JE, Clarke LA. The International Consensus Panel on the management and treatment of Mucopolysaccharidosis I. Pediatrics. 2009;123:19-29.

6. Pastores G, Arn P, Beck M, Clarke JT, Guffon N, Kaplan P, et al. The MPS I registry: Design, methodology, and early findings of a global disease registry for monitoring patients with mucopolysaccharidosis type I. Mol Genet Metab. 2007;91:37-47.

7. Diagnosis and treatment trends in mucopolysaccharidosis I: findings from the MPS I Registry Kristin D'Aco, Lisa Underhill, Lakshmi Rangachari, Pamela Arn, Gerald F. Cox, Roberto Giugliani, Torayuki Okuyama, Frits Wijburg, Paige 
Kaplan Eur J Pediatr. 2012 Jun; 171(6):

911-919. Published online 2012 Jan 11. doi: 10.1007/s00431-011-1644xPMCID:PMC3357468

8. Muenzer J, Wraith JE, Clarke LA, International Consensus Panel on Management and Treatment of Mucopolysaccharidosis I. Pediatrics. 2009 Jan; 123(1):19-29.

9. Enzyme replacement therapy with laronidase (Aldurazyme (®)) for treating mucopolysaccharidosis type I. [Cochrane Database Syst Rev. 2013]

10. Human genome-edited hematopoietic stem cells phenotypically correct Mucopolysaccharidosis type I.Gomez-Ospina N, Scharenberg SG, Mostrel N, Bak RO, Mantri S, Quadros RM, Gurumurthy CB, Lee C, Bao G, Suarez CJ, et al. Nat Commun. 2019 Sep 6; 10(1): 4045. Epub 2019 Sep 6.

11. Therapeutic Options for Mucopolysaccharidoses: Current and Emerging Treatments. Sawamoto K, Stapleton M, Alméciga-Díaz CJ, Espejo-Mojica AJ, Losada JC, Suarez DA, Tomatsu S. Drugs. 2019 Jul; 79(10):1103-1134.

12. Taiwan National Newborn Screening Program by Tandem Mass Spectrometry for Mucopolysaccharidoses Types I, II, and VI. Chan MJ, Liao HC, Gelb MH, Chuang CK, Liu MY, Chen HJ, Kao SM, Lin HY, Huang YH, Kumar AB, et al. J Pediatr. 2019 Feb; 205:176-182. Epub 2018 Nov 6. 